\title{
Experimental avian paramyxovirus serotype-3 infection in chickens and turkeys
}

\author{
Sachin Kumar $^{1}$, Flavia Militino Dias ${ }^{1}$, Baibaswata NAYAK ${ }^{1}$, Peter L. Collins ${ }^{2}$, \\ Siba K. SAMAL ${ }^{1 *}$ \\ ${ }^{1}$ Virginia-Maryland Regional College of Veterinary Medicine, University of Maryland, College Park, \\ MD 20742, Maryland, USA \\ ${ }^{2}$ Laboratory of Infectious Diseases, National Institute of Allergy and Infectious Diseases, \\ National Institute of Health, Bethesda, USA
}

(Received 15 June 2010; accepted 20 July 2010)

\begin{abstract}
Avian paramyxoviruses (APMV) are divided into nine serotypes. Newcastle disease virus (APMV-1) is the most extensively characterized, while relatively little information is available for the other APMV serotypes. In the present study, we examined the pathogenicity of two divergent strains of APMV-3, Netherlands and Wisconsin, in (i) 9-day-old embryonated chicken eggs, (ii) 1-day-old specific pathogen free (SPF) chicks and turkeys, and (iii) 2-week-old SPF chickens and turkeys. The mean death time in 9-day-old embryonated chicken eggs was $112 \mathrm{~h}$ for APMV-3 strain Netherlands and $>168 \mathrm{~h}$ for strain Wisconsin. The intracerebral pathogenicity index in 1-day-old chicks for strain Netherlands was 0.39 and for strain Wisconsin was zero. Thus, both strains are lentogenic. Both the strains replicated well in brain tissue when inoculated intracerebrally in 1-day-old SPF chicks, but without causing death. Mild respiratory disease signs were observed in 1-day-old chickens and turkeys when inoculated through oculonasal route with either strain. There were no overt signs of illness in 2-weeks-old chickens and turkeys by either strain, although all the birds seroconverted after infection. The viruses were isolated predominantly from brain, lungs, spleens, trachea, pancreas and kidney. Immunohistochemistry studies also showed the presence of large amount of viral antigens in both epithelial and sub-epithelial lining of respiratory and alimentary tracts. Our result suggests systemic spread of APMV-3 even though the viral fusion glycoprotein does not contain the canonical furin proteases cleavage site. Furthermore, there was little or no disease despite systemic viral spread and abundant viral replication in all the tissues tested.
\end{abstract}

\section{avian paramyxovirus / pathogenicity}

\section{INTRODUCTION}

Members of the family Paramyxoviridae are pleomorphic enveloped particles that contain a single-stranded, non-segmented RNA genome of 13-19 $\mathrm{kb}$ [20]. These viruses have been isolated from many species of avian, terrestrial and aquatic animals around the world. Some members of the family Paramyxoviridae are well

\footnotetext{
* Corresponding author: ssamal@umd.edu
}

known human (measles, mumps, respiratory syncytial virus and various human parainfluenza viruses) and animal (rinderpest virus, canine distemper virus and Newcastle disease virus (NDV)) pathogens, while the pathogenic potential of many others is still unknown. The family is divided into two subfamilies, Paramyxovirinae and Pneumovirinae. Subfamily Paramyxovirinae is further divided into five genera: Rubulavirus, Respirovirus, Henipavirus, Morbillivirus and Avulavirus. Subfamily

This is an Open Access article distributed under the terms of the Creative Commons Attribution-Noncommercial License (http://creativecommons.org/licenses/by-nc/3.0/), which permits unrestricted use, distribution, and reproduction in any noncommercial medium, provided the original work is properly cited. 
Pneumovirinae is divided into two genera: Pneumovirus and Metapneumovirus [23].

All paramyxoviruses that have been isolated to date from avian species are placed in the genus Avulavirus except for avian metapneumoviruses that are placed in the genus Metapneumovirus due to antigenic and genomic organization differences. The avian paramyxoviruses (APMV) that comprise genus Avulavirus have been divided into nine different serotypes (APMV-1 through -9) based on haemagglutination inhibition (HI) and neuraminidase inhibition (NI) assays [6]. APMV-1 comprises all strains of NDV and is the most completely characterized serotype due to the severity of disease caused by virulent NDV strains in chickens [3]. The complete genome sequences and the molecular determinants of virulence have been determined for representative NDV strains [13, 15, 17, 28, 31]. As a first step in characterizing the other APMV serotypes, complete genome sequences of one or more representatives of APMV serotypes 2 to 9 were recently determined, expanding our knowledge about these viruses [12, 18, 26, 27, 34, 38, 42].

NDV causes respiratory, neurological or enteric disease in birds. Among poultry, chickens are the most susceptible while geese and ducks are the least susceptible [40]. NDV strains are classified into low virulent (lentogenic), moderately virulent (mesogenic), and highly virulent (velogenic) pathotypes based on pathogenicity in chickens. In contrast very little is known on pathogenicity of APMV-2 to -9 in chickens and turkeys. APMV-2 has been shown to cause mild disease and drop in egg production in chickens and turkeys [41]. APMV-6 and -7 have been associated with respiratory disease in turkeys $[33,35]$. APMV-4, -8 and -9 have been isolated from different species of birds but the clinical signs of the disease in those birds were not apparent $[5,11,14,22,37]$. Recently, experimental infection of 1-day-old chicks with APMV-2, -4 and -6 showed viral infection in gastrointestinal tract, respiratory tract and pancreas [41].

APMV-3 has been isolated from wild and domesticated birds in different parts of the world [1, 3, 21, 39]. Recently, APMV-3 was isolated from ostrich, indicating a wide host range for the virus [16]. The virus has been isolated from diseased turkeys associated with coughing, nasal discharge and swelling of the infra-orbital sinus [29]. APMV-3 has been associated with encephalitis and high mortality in caged birds [39]. The virus causes acute pancreatitis and central nervous system symptoms in Psittacine and Passerine birds [10]. APMV-3 also infects chickens at an early age, with evidence of stunting growth that may be more marked in broiler chicken breeds [4]. In terms of pathogenicity to domestic poultry birds, APMV-3 probably is second in importance to NDV. The exact economic impact of APMV-3 infection in poultry industry is not known. This is partly because the pathogenicity of APMV-3 in poultry species is not well studied. There is a high degree of amino acid sequence variation between APMV-3 and APMV-1 (NDV), but by the HI test there is cross reaction between APMV-3 and APMV-1 serum samples, which often leads to misdiagnosis of APMV-3 as APMV-1.

Currently, it is not known whether there is any variation in pathogenicity among APMV-3 strains in domestic poultry birds. The purpose of this study was to evaluate the pathogenicity of APMV-3 strains Netherlands and Wisconsin in chickens and turkeys. Both of these strains were completely sequenced recently $[18,19]$. APMV-3 strain Wisconsin was first isolated in 1968 in Wisconsin from a turkey flock showing coughing, depression, drop in egg production and anorexia [39]. APMV-3 strain Netherlands was isolated in 1975 from a parakeet showing nervous disease symptoms similar to NDV in the Netherlands and is the prototype for the entire serotype [2, 36]. Initially, these two viruses were considered as two different strains of APMV-3 based on cross HI test using monoclonal antibodies [8, 9]. Recently, reciprocal cross HI and cross neutralization assays using post infection serum from chicken indicated that these two strains are antigenically distinct, although the difference was modest [19]. However, complete genome sequence analysis revealed substantial genome-wide nucleotide and amino acid sequence differences that are consistent with the two strains representing distinct antigenic subgroups $[18,19]$. In the present study, we studied infection of APMV-3 strains 
Wisconsin and Netherlands in 9-day-old embryonated chicken eggs, 1-day-old chicks and turkeys, and 2-week-old chickens and turkeys in order to investigate their tropism and pathogenicity. Birds were infected by the oculonasal route and the viral tropism and replication efficiency were evaluated by quantitative virology and immunohistochemistry (IHC) of a wide range of possible target organs. In addition, a separate group of 1-day-old chicks were infected intracerebrally to evaluate the potential of these viruses to replicate in neural cells.

\section{MATERIALS AND METHODS}

\subsection{Viruses and cells}

APMV-3 strains parakeet/Netherlands/449/75 and turkey/Wisconsin/68 (obtained from National Veterinary Service Laboratory, Ames, Iowa, USA) and the NDV mesogenic strain Beaudette C (BC) and lentogenic strain LaSota were propagated in 9-day-old specific pathogen free (SPF) embryonated chicken eggs via allantoic route of inoculation. Work with NDV$\mathrm{BC}$ was performed in our Bio Safety Level (BSL)-3 animal facility. The allantoic fluids from infected embryonated eggs were collected $96 \mathrm{~h}$ post-inoculation and virus titer was determined by hemagglutination (HA) assay with $0.5 \%$ chicken RBC. The virus titer in infected tissue samples was determined by the tissue culture infective dose $\left(\mathrm{TCID}_{50}\right)$ [30] method and by plaque assay in chicken embryo fibroblast (DF-1) cells (ATCC, Manassas, VA, USA). DF-1 cells were maintained in Dulbecco's minimum essential medium (DMEM) with $10 \%$ fetal bovine serum (FBS) at $37{ }^{\circ} \mathrm{C}$ with $5 \% \mathrm{CO}_{2}$ [18]. For the plaque assay, DF-1 cell monolayers in 12 well plates were infected with different dilutions of tissue homogenates. The tissue homogenates were allowed to adsorb for $1 \mathrm{~h}$ at $37{ }^{\circ} \mathrm{C}$, washed with phosphate-buffered saline (PBS), and then overlaid with $1 \mathrm{~mL}$ DMEM containing $0.8 \%$ (wt/vol) methylcellulose. The virus plaques in the DF-1 cell monolayer were visualized $96 \mathrm{~h}$ PI after staining with $1 \%$ crystal violet.

\subsection{Pathogenicity index tests}

The pathogenicity of the APMV-3 strains Netherlands and Wisconsin was determined by two standard pathogenicity tests. These included the mean death time (MDT) in 9-day-old embryonated SPF chicken eggs and the intracerebral pathogenicity index (ICPI) in 1-day-old SPF chicks ${ }^{1}$.

MDT value was determined following the standard procedure [7]. Briefly, a series of 10 -fold $\left(10^{-6}\right.$ to $10^{-12}$ ) dilutions of fresh infective allantoic fluid in sterile PBS were made and $0.1 \mathrm{~mL}$ of each dilution was inoculated into the allantoic cavities of five 9-day-old embryonated SPF chicken eggs, which were then incubated at $37^{\circ} \mathrm{C}$. Each egg was examined three times daily for 7 days, and the times of embryo deaths were recorded. The minimum lethal dose is the highest virus dilution that caused death of all the embryos. MDT is the mean time in hours for the minimum lethal dose to kill all inoculated embryos. The MDT has been used to characterize the NDV pathotypes as follows: velogenic (less than $60 \mathrm{~h}$ ), mesogenic (60 to $90 \mathrm{~h}$ ), and lentogenic (more than $90 \mathrm{~h}$ ) [3].

For determining ICPI value, $0.05 \mathrm{~mL}$ of $1: 10$ dilution of fresh infective allantoic fluid $\left(2^{8} \mathrm{HA}\right.$ units) of each virus was inoculated into groups of 10 one-day-old SPF chicks via the intracerebral route. The birds were observed for clinical symptoms and mortality every $8 \mathrm{~h}$ for a period of 8 days. At each observation, the birds were scored as follows: 0 , healthy; 1, sick; and 2, dead. The ICPI is the mean score for all of the birds in the group over the 8-day period. Highly virulent NDV (velogenic) viruses give values approaching 2 and avirulent NDV (lentogenic) viruses give values close to 0 [7].

\subsection{Replication and viral growth kinetics in brain tissue of 1-day-old chicks}

To determine the ability of APMV-3 strains Netherlands and Wisconsin to replicate in chicken brain tissue, groups of 15 one-day-old SPF chicks were inoculated with a dose of $0.05 \mathrm{~mL}$ of a 1:10 dilution of $2^{8}$ HA units of fresh infected allantoic fluid via the intracerebral route. NDV strain BC and LaSota were included for comparison purposes. Brain samples were collected by sacrificing three birds from each group at 1, 2, 3, 4 and 5 days post inoculation or as and when the birds died of infection. The virus titers in the tissue samples were determined by $\mathrm{TCID}_{50}$ in DF-1 cells [30].

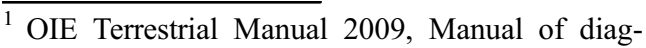
nostic tests and vaccines for terrestrial animals 2009, http://www.oie.int/fr/normes/mmanual/2008/ pdf/2002.2003.2014_NEWCASTLE_DIS.pdf. 


\subsection{Pathogenesis assessment in chickens and turkeys}

1-day-old and 2-week-old SPF chickens (Charles River, North Franklin, Connecticut, USA) and 1-day-old and 2-week-old turkeys (Murry-McMurry hatchery, Webster City, Iowa, USA) were housed in positive pressure isolators in our BSL-2 facility. Birds confirmed to be negative for APMV-3 specific antibody by $\mathrm{HI}$ assay were further isolated for pathogenesis experiments and housed in negative pressure isolators. The 1-day-old and 2-week-old chickens and turkeys, in groups of 12 for each species and age, were infected with $0.1 \mathrm{~mL}\left(10^{3} \mathrm{PFU}\right)$ per bird of APMV-3 strain Netherlands or Wisconsin through the occulonasal route. Infections with the different strains were performed at separate times to avoid cross infections. An additional six birds of each species/age group remained as uninfected controls and sacrificed 14 day post infection (DPI) after collection of serum. Birds were provided with food and water ad libitum and monitored daily for any visible signs and symptoms twice daily. Three birds from each infected group were euthanized on 3, 5, 7 and 14 DPI by rapid asphyxiation in a $\mathrm{CO}_{2}$ chamber. The birds were swabbed orally and cloacally just before euthanasia. The following tissue samples were collected both for IHC and for virus isolation: brain, trachea, lung, spleen, kidney and pancreas. In addition serum samples were collected on day 14 when the three remaining birds in each group and the control birds were euthanized. Seroconversion was evaluated by HI assay [1].

\subsection{Virus detection and quantification from tissue samples and swabs}

Half of the tissue samples containing brain, trachea, lung, spleen, kidney and pancreas were collected aseptically in DMEM in 10x antibiotic solution containing Penicillin/Streptomycin/Amphotericin B to determine virus content. Briefly, a 10\% homogenate of the tissue samples were prepared by using a homogenizer and centrifuged at $420 \times g$ for $10 \mathrm{~min}$. The virus content in the supernatants of tissue homogenates was determined by plaque assay in DF-1 cells. Values for each tissue sample were based on average plaque count from two wells. The other half of the tissue samples were flash-frozen on dry ice using chilled 2-methyl butane, held for approximately $24 \mathrm{~h}$, and processed for IHC.

The oral and cloacal swabs were collected in $1 \mathrm{~mL}$ of DMEM containing antibiotics. The swab containing tubes were centrifuged at $1000 \mathrm{~g}$ for $20 \mathrm{~min}$, and the supernatant was removed and flash frozen on dry ice for subsequent virus isolation. Virus isolation was performed by infecting the supernatant into 9-day-old SPF embryonated chicken eggs. Positive samples were identified by HA activity of the allantoic fluid harvested from eggs four days postinoculation. In addition the virus titers in the positive swab samples were determined using the $\mathrm{TCID}_{50}$ method and DF-1 cells [30].

\subsection{IHC}

Tissue samples collected in chilled 2-methyl butane were sectioned by using optimal cutting temperature compound under cryostat (Dolbey-Jamison, Pottstown, Pennsylvania, USA). The sections were immunostained to detect viral phosphoprotein $(\mathrm{P})$ using the following protocol. Briefly, the frozen sections were thawed and rehydrated in three changes of PBS (10 min each). The sections were fixed in ice cold acetone for $15 \mathrm{~min}$ at $-80{ }^{\circ} \mathrm{C}$ and then washed three times with $2 \%$ BSA in PBS and blocked with the same solution for $1 \mathrm{~h}$ at room temperature. The sections were then incubated with a 1:500 dilution of the primary antibody (antipeptide polyclonal antisera raised against the $P$ proteins of APMV-3 strains Netherland and Wisconsin in rabbits) in PBS overnight in a humidified chamber. After three washes with $2 \%$ BSA in PBS, sections were incubated with the secondary antibody (FITC conjugated goat antirabbit antibody) for $30 \mathrm{~min}$. After a further wash cycle, the sections were mounted with glycerol and viewed under a fluorescence microscope.

\subsection{Preparation of antipeptide polyclonal antisera against the APMV-3 $P$ proteins in rabbits}

The antipeptide antisera to the $\mathrm{P}$ proteins of the two APMV-3 strains were generated using the following protocol. Peptides DKTPDQGQPSATPS (corresponding to 60 to 73 amino acid (aa) of P protein of strain Netherlands), SPSQSSSPSPEPST, and FYIPKVNNYHSN (corresponding to 39-52 and 99-110 aa, respectively, of $P$ protein of strain Wisconsin) were custom synthesized (Invitrogen, Carlsbad, California, USA). $0.2 \mathrm{mg}$ of each protein was mixed in complete Freund's adjuvant and injected subcutaneously into a rabbit. After two weeks a booster immunization was given with the respective $0.2 \mathrm{mg}$ of protein in incomplete Freund's adjuvant and two weeks later the hyperimmune serum was collected. The antisera were tested by Western blot analysis and were found to recognize specifically the $\mathrm{P}$ protein of their respective APMV-3 strain (data not shown). 


\section{RESULTS}

\subsection{Pathogenicity index tests}

The pathogenicity of APMV-3 strains Netherlands and Wisconsin was evaluated by MDT in 9-day-old embryonated chicken eggs and ICPI in 1-day-old-chicks. The lentogenic NDV strain LaSota was included in the pathogenicity tests for comparison. The MDT for APMV-3 strain Netherland was $112 \mathrm{~h}$ while that of APMV-3 strain Wisconsin was $>168 \mathrm{~h}$. The ICPI value for APMV-3 strain Netherlands was 0.39 while the ICPI for strain Wisconsin was zero. The MDT and ICPI values of NDV strain LaSota were $110 \mathrm{~h}$ and zero, respectively, consistent with a lentogenic virus. Although, the ICPI and MDT values of strain Netherlands were higher than those of strain Wisconsin; their values indicate that both the APMV-3 strains are lentogenic viruses. These results indicate that APMV-3 strain Netherlands and Wisconsin are probably nonpathogenic to chickens, similar to lentogenic NDV strains.

\subsection{Virus growth in the chicken brain}

The ability of the APMV-3 strains Netherlands and Wisconsin to grow in the brains of 1-day-old chicks was evaluated in parallel with the mesogenic neurotropic NDV strain BC. This study was performed to determine whether the 0.39 and zero ICPI values of the APMV-3 strains Netherlands and Wisconsin, respectively, was due to the inability of the viruses to grow intracerebrally or if there was virus multiplication without a high degree of cell destruction.

Virus replication was evaluated by inoculating $0.05 \mathrm{~mL}$ of $1: 10$ dilution of $2^{8}$ HAU of each of the four viruses, strains Netherlands, Wisconsin, $\mathrm{BC}$ and LaSota, into the brains of 15 one-dayold SPF chicks per group. Three birds from each group were sacrificed on days 1, 2, 3, 4 and 5 post inoculation and virus titers in brain tissue were assayed and expressed as TCID 50 per gram of the brain in DF-1 cells (Fig. 1). Neither of the two APMV-3 strains produced any clinical signs nor did they kill any chicks during the 5-day time course. Both of the strains of APMV-3 were iso- lated from the brain homogenates of all of the chicks on days 1 to 5 day post inoculation, grew with similar kinetics, and reached similar maximum titers of $2 \times 7.9 \log _{10}$ and $2 \times 7.6 \log _{10}$ $\mathrm{TCID}_{50} / \mathrm{g}$ on day 5 for strains Netherlands and Wisconsin, respectively (Fig. 1). In comparison, NDV strain BC replicated more rapidly, reached a titer of $2 \times 8.25 \log _{10}$ TCID $_{50} / g$ of brain tissue on day 3 post inoculation, and killed all of the chicks by day 3, while strain LaSota showed no replication. The results showed that the efficiency of virus replication of the two APMV-3 strains in brain tissue was similar. Interestingly, while the two APMV-3 strains replicated more slowly than NDV-BC, they achieved a similar final titer compared to NDV-BC but without causing noticeable neurological disease and with no mortality.

\subsection{Experimental infection of SPF chickens and turkeys: pathology}

1-day-old and 2-week-old chickens and turkeys were inoculated with either APMV-3 strain by the occulonasal route, and birds from each group were sacrificed on days $3,5,7$, and 14 and evaluated for pathology, virus shedding, and virus replication in selected tissues. Infection of either APMV-3 strain in 1-day-old chickens and turkeys resulted in mild clinical symptoms that included altered gait, respiratory distress, dullness, ruffled-feathers, loss of appetite and weight loss. The birds of the 1-day-old groups of both species generally started showing symptoms at 3 DPI and recovered after 7 DPI. The other visible signs included slight diarrhea that was evident in 1-day-old chickens and turkeys at 4 DPI. The neurological symptoms were more evident in all 1-day-old chicks and turkeys infected with APMV-3 strain Netherlands as compared to strain Wisconsin. In contrast, infection of 2-week-old chickens and turkeys with the APMV-3 strains did not result in any clinical signs of disease. APMV-3 infection did not kill any of the 1-day-old or 2-week-old chickens and turkeys (Tabs. I and II). The most remarkable finding upon postmortem examination of birds was enlargement of the pancreas with focal necrosis at 5 DPI in the 2-week-old chickens and turkeys (Fig. 2). The foci of necrosis were distributed along the entire length of pancreas 


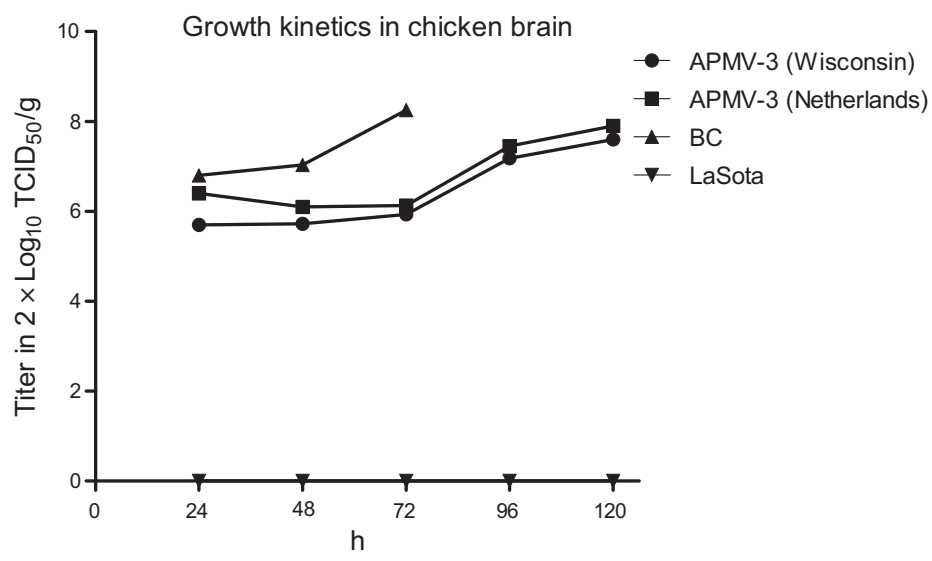

Figure 1. Growth kinetics of APMV-3 strain Netherlands and Wisconsin in the brains of 1-day-old chicks in comparison with neurotropic APMV-1 strain Beaudette C (BC) and lentogenic strain LaSota. Fifteen 1-day-old chicks were inoculated with $0.05 \mathrm{~mL}$ of 1:10 dilution of $2^{8} \mathrm{HA}$ units of APMV-1 strain BC or APMV-1 strain LaSota or APMV-3 strain Netherlands or Wisconsin via the intracerebral route. Brain tissue was collected by sacrificing three birds from each group on 1,2, 3, 4 and 5 days post inoculation, except that in the case of NDV BC all remaining birds died of infection on day 3 and were assayed at that time point. Each time point represents the geometric mean of the individual virus titers of three birds.

Table I. Virus shedding in 1-day-old and 2-week-old chickens infected with APMV-3. Virus shedding in oral secretions (O) and cloacal swabs (C) were measured for three birds from each group on the indicated days. Mean body weight was also measured at sacrifice. Sera were collected from three birds in each group on day 14 and mean APMV-3-specific HI titers were determined. The pre-infection and control HI titers were 2 or less.

\begin{tabular}{|c|c|c|c|c|c|c|}
\hline \multirow[t]{2}{*}{ Virus } & \multicolumn{4}{|c|}{ Virus shedding } & \multirow{2}{*}{$\begin{array}{l}\text { Mean body } \\
\text { weight }(\mathrm{g})\end{array}$} & \multirow{2}{*}{$\begin{array}{c}\text { HI titer } \\
\log 214 \text { DPI }\end{array}$} \\
\hline & $\begin{array}{c}\text { 3rd day } \\
\mathrm{O} / \mathrm{C}\end{array}$ & $\begin{array}{c}\text { 5th day } \\
\mathrm{O} / \mathrm{C}\end{array}$ & $\begin{array}{c}\text { 7th day } \\
\mathrm{O} / \mathrm{C}\end{array}$ & $\begin{array}{c}\text { 14th day } \\
\mathrm{O} / \mathrm{C}\end{array}$ & & \\
\hline \multicolumn{7}{|c|}{ 1-day-old chickens infected with APMV-3 } \\
\hline APMV-3 Netherlands & $+/-(3 / 0)$ & $+/-(3 / 0)$ & $-1-$ & $-1-$ & $70 \pm 0.98$ & $1: 128$ \\
\hline APMV-3 Wisconsin & $+/-(3 / 0)$ & $+/-(2 / 0)$ & $-1-$ & $-1-$ & $75 \pm 1.02$ & $1: 128$ \\
\hline Control & $-1-$ & $-1-$ & $-1-$ & $-1-$ & $80 \pm 1.04$ & $\mathrm{~N} / \mathrm{D}$ \\
\hline \multicolumn{7}{|c|}{ 2-week-old chickens infected with $A P M V-3$} \\
\hline APMV-3 Netherlands & $+/-(3 / 0)$ & $-1-$ & $-1-$ & $-1-$ & $180 \pm 0.78$ & $1: 512$ \\
\hline APMV-3 Wisconsin & $-1-$ & $-1-$ & $-1-$ & $-1-$ & $175 \pm 0.92$ & $1: 256$ \\
\hline Control & $-1-$ & $-1-$ & $-1-$ & $-1-$ & $180 \pm 1.03$ & $\mathrm{~N} / \mathrm{D}$ \\
\hline
\end{tabular}

and the extent of necrosis was similar in both chickens and turkeys. Gross examination of other organs including the brain, trachea, liver, kidney, spleen and lung showed normal tissue morphology with no noticeable gross lesions in either the 1-day-old or 2-week-old birds.

\subsection{Experimental infection of SPF chickens and turkeys: virus isolation and titration in oral and cloacal swabs}

From the experiment above, oral and cloacal swabs were taken when birds were sacrificed on 
Table II. Virus shedding in 1-day-old and 2-week-old turkeys infected with APMV-3. Virus shedding in oral secretions $(\mathrm{O})$ and cloacal swabs $(\mathrm{C})$ were measured for three birds from each group on the indicated days. Mean body weight was also measured at sacrifice. Sera were collected from three birds in each group on day 14 and mean APMV-3-specific HI titers were determined. The pre-infection and control HI titers were 2 or less.

\begin{tabular}{|c|c|c|c|c|c|c|}
\hline \multirow[t]{2}{*}{ Virus } & \multicolumn{4}{|c|}{ Virus shedding } & \multirow{2}{*}{$\begin{array}{l}\text { Mean body } \\
\text { weight }(\mathrm{g})\end{array}$} & \multirow{2}{*}{$\begin{array}{c}\text { HI titer } \\
\log 214 \mathrm{DPI}\end{array}$} \\
\hline & $\begin{array}{c}\text { 3rd day } \\
\text { O/C }\end{array}$ & $\begin{array}{c}\text { 5th day } \\
\mathrm{O} / \mathrm{C}\end{array}$ & $\begin{array}{l}\text { 7th day } \\
\text { O/C }\end{array}$ & $\begin{array}{l}\text { 14th day } \\
\text { O/C }\end{array}$ & & \\
\hline \multicolumn{7}{|c|}{ 1-day-old turkeys infected with APMV-3 } \\
\hline APMV-3 Netherlands & $+/-(2 / 0)$ & $+/-(2 / 0)$ & $-1-$ & $-1-$ & $80 \pm 0.95$ & $1: 128$ \\
\hline APMV-3 Wisconsin & $+/-(2 / 0)$ & $+/-(2 / 0)$ & $-1-$ & $-1-$ & $90 \pm 0.75$ & $1: 128$ \\
\hline Control & $-1-$ & $-1-$ & $-1-$ & $-1-$ & $120 \pm 0.96$ & $\mathrm{~N} / \mathrm{D}$ \\
\hline \multicolumn{7}{|c|}{ 2-week-old turkeys infected with $A P M V$-3 } \\
\hline APMV-3 Netherlands & $+/-(2 / 0)$ & $-1-$ & $-1-$ & $-1-$ & $140 \pm 1.02$ & $1: 256$ \\
\hline APMV-3 Wisconsin & $-1-$ & $-1-$ & $-1-$ & $-1-$ & $170 \pm 0.99$ & $1: 128$ \\
\hline Control & $-1-$ & $-1-$ & $-1-$ & $-1-$ & $180 \pm 0.92$ & N/D \\
\hline
\end{tabular}

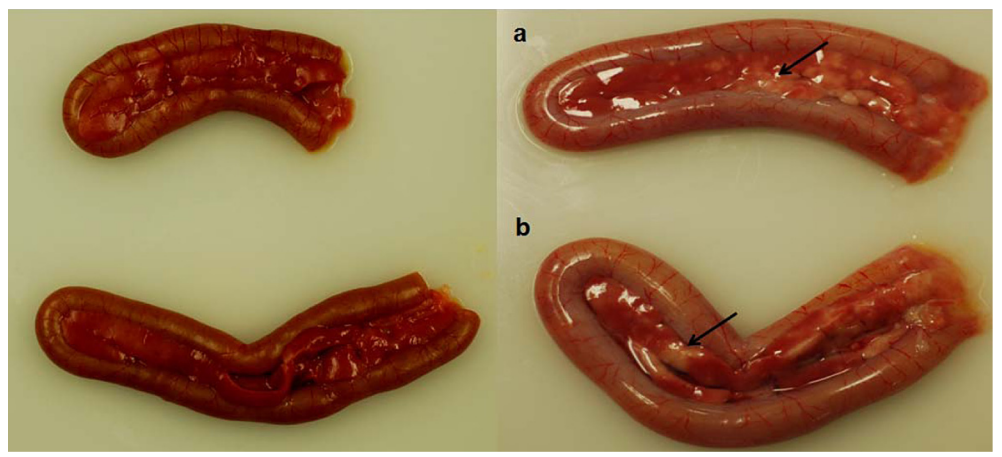

Figure 2. Gross morphology of pancreas collected from 2-week-old turkeys infected with APMV-3 strain Netherlands (a) or Wisconsin (b): comparison with pancreas from uninfected controls (left panels) reveals necrotic foci along the length of the infected organ. (A color version of this figure is available at www.vetres.org.)

days 3, 5, 7 and 14 DPI. These samples were inoculated into embryonated chicken eggs to detect the presence of infectious virus, which was determined by HA assay of egg allantoic fluid (Tabs. I and II). Samples that were positive for virus were analyzed for virus quantitation by plaque assay in DF-1 cells (data not shown). Neither APMV-3 strain was isolated from cloacal swabs from any of the infected birds. Virus was isolated from oral swabs from 1-day-old chickens on days 3 and 5 for both strains, and from 2-week-old chickens on day 3 for strain Netherlands only (Tab. I). This same pattern was observed for virus isolated from oral swabs from 1-day-old and 2-week-old turkeys (Tab. II). Thus, strain Wisconsin was not shed from any of the 2-week-old chickens or turkeys. The highest titers in chickens (2.15 $\log _{10}$ $\mathrm{TCID}_{50} / \mathrm{mL}$ ) were observed in oral swabs collected on 3 DPI from 1-day-old chicks infected with either strain of APMV-3 (data not shown). Similarly, in turkeys, the highest titers $(2.12$ 


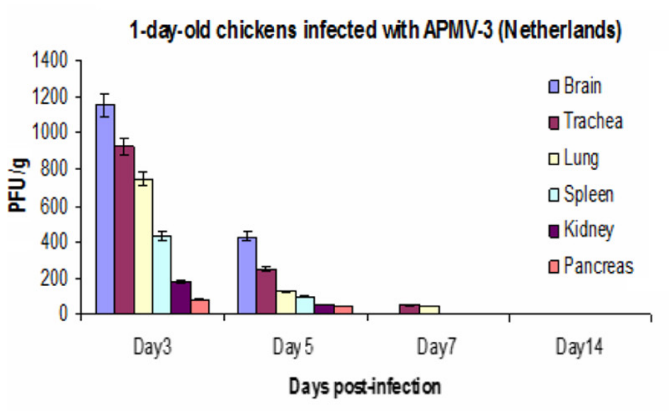

2-week-old chickens infected with APMV-3 (Netherlands)

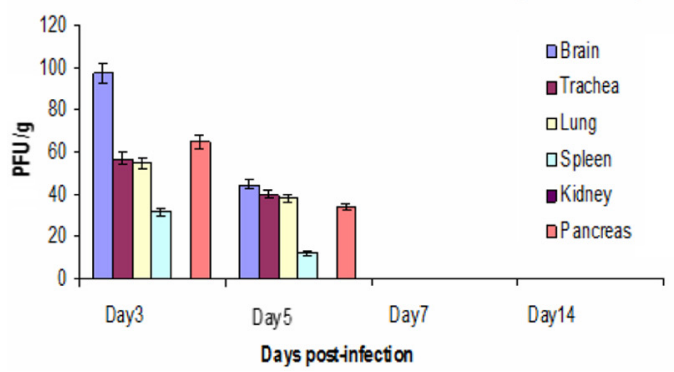

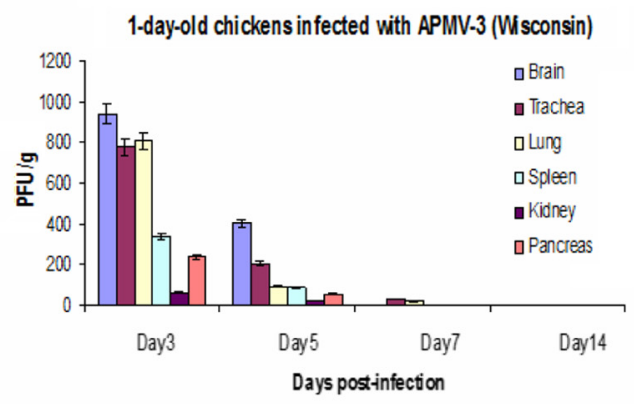

2-week-old chickens infected with APMV-3 (Wisconsin)

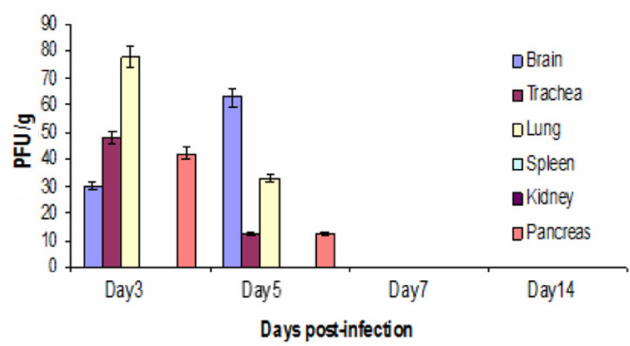

Figure 3. Virus titers from the indicated organs on the indicated days after infection of 1-day-old (upper panels) or 2-week-old (lower panels) chickens with APMV-3 strain Netherlands (left panels) or Wisconsin (right panels). Titers are shown as mean PFU/g. (A color version of this figure is available at www.vetres.org.)

$\log _{10} \mathrm{TCID}_{50} / \mathrm{mL}$ ) were observed in 1-day-old poults at 3 DPI with either strain of APMV-3 (data not shown).

\subsection{Experimental infection of SPF chickens and turkeys: virus isolation and titration in tissue samples}

In the experiment described above, the following tissue samples were collected from three birds per group on 3, 5, 7 and 14 DPI: brain, trachea, lung, spleen, kidney and pancreas. Homogenates of these tissues were inoculated into embryonated chicken eggs to detect the presence of infectious virus, as described above, and positive samples were titrated by plaque assay in DF-1 cells.

Figure 3 shows the distribution and titers of the two APMV-3 strains in various organs on different DPI of chickens. Infection with APMV-3 strains Netherlands and Wisconsin in 1-day-old chicks and 2-week-old chickens resulted in efficient replication of both viruses in the various organs tested. In general, both viruses replicated in most of the tested organs at early time periods of infection and were cleared at 7 DPI. The virus replication titers were higher in 1-day-old chicks than in 2-weeks-old chickens, and replication usually was the highest in the brain, trachea, and lung. Although APMV-3 strain Wisconsin replicated less efficiently in 2-week-old chickens, the titer of virus in lungs on day 1 was somewhat higher than that of strain Netherlands, suggesting that it might be more respirotropic, whereas strain Netherlands appeared to be more neurotropic on day 1 in the 2 -week-old birds.

In general, infected turkeys had virus replication in fewer organs and for shorter duration than chickens of same age groups (Fig. 4). Strain Netherlands was isolated from all of the tested organs on 3 DPI except for the pancreas in 1-day-old turkeys and the kidneys in 


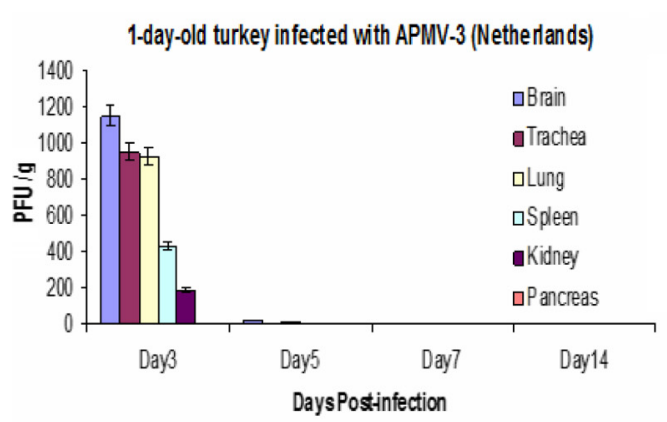

2-week-old turkey infected with APMV-3 (Iletherlands)

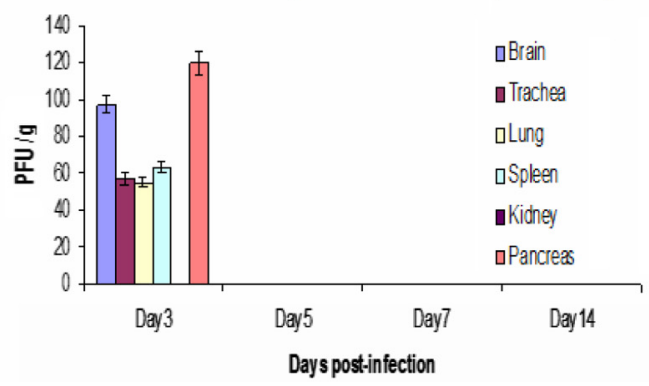

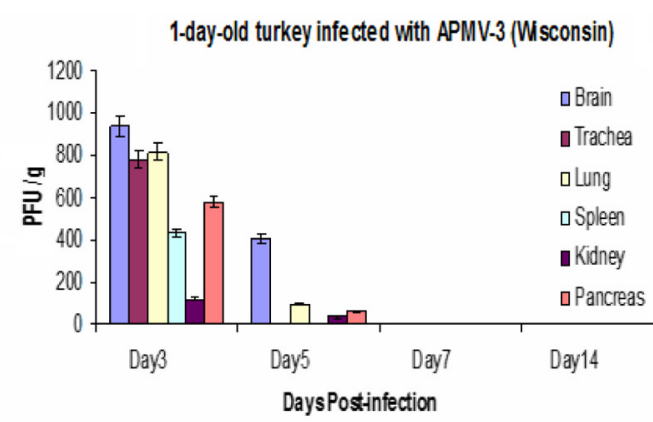

2-week-old turkey infected with APMV-3 (Wisconsin)

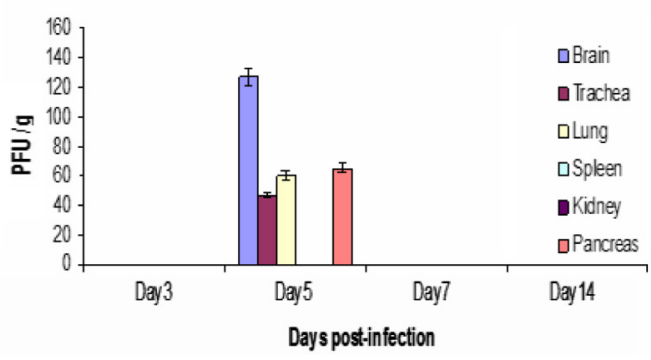

Figure 4. Virus titers from the indicated organs on the indicated days after infection of 1-day-old (upper panels) or 2-week-old (lower panels) turkeys with APMV-3 strain Netherlands (left panels) or Wisconsin (right panels). Titers are shown as mean PFU/g. (A color version of this figure is available at www.vetres.org.)

2-week-old turkeys. Strain Wisconsin was isolated from all of the tested organs on 3 DPI. Interestingly, APMV-3 strain Wisconsin was not detected at all on 3 DPI in 2-week-old turkeys, but was detected in some of the organs on 5 DPI (Fig. 4). No virus of either strain was detectable on 5 and 14 DPI (Fig. 4) from any of the tissues harvested from the infected turkeys, indicating virus clearance, whereas complete clearance in chickens did not occur until 14 DPI. These results suggest that turkeys are less susceptible to infection with either APMV-3 strain compared to chickens.

\subsection{Seroconversion}

An HI assay using chicken erythrocytes was performed with the sera collected from chickens and turkeys of both age groups on 14 DPI. The HI titers of the pre-infection and control chickens and turkeys were 2 or less. An HI titer of greater than 8 was considered positive. Each of the 1-day-old and 2-week-old chickens and turkeys infected with either strain seroconverted. The mean HI titers in 1-day-old chicks for both the strains were 1:128; while the mean HI titers in 2-week-old chickens for strain Netherlands and Wisconsin were 1:512 and 1:256 respectively (Tab. I). Similarly, the mean HI titers in 1-day-old-turkeys for the both the strains were 1:128, while the mean HI titers in 2-weeks-old turkeys for strain Netherlands and Wisconsin were $1: 256$ and 1:128 respectively (Tab. II).

\subsection{IHC}

The frozen sections of all the virus-positive tissue samples and some of the virus-negative control samples were immunostained using antipeptide polyclonal antibodies against the P proteins of APMV-3 strains Netherlands and Wisconsin. Large amounts of viral $\mathrm{P}$ antigen 

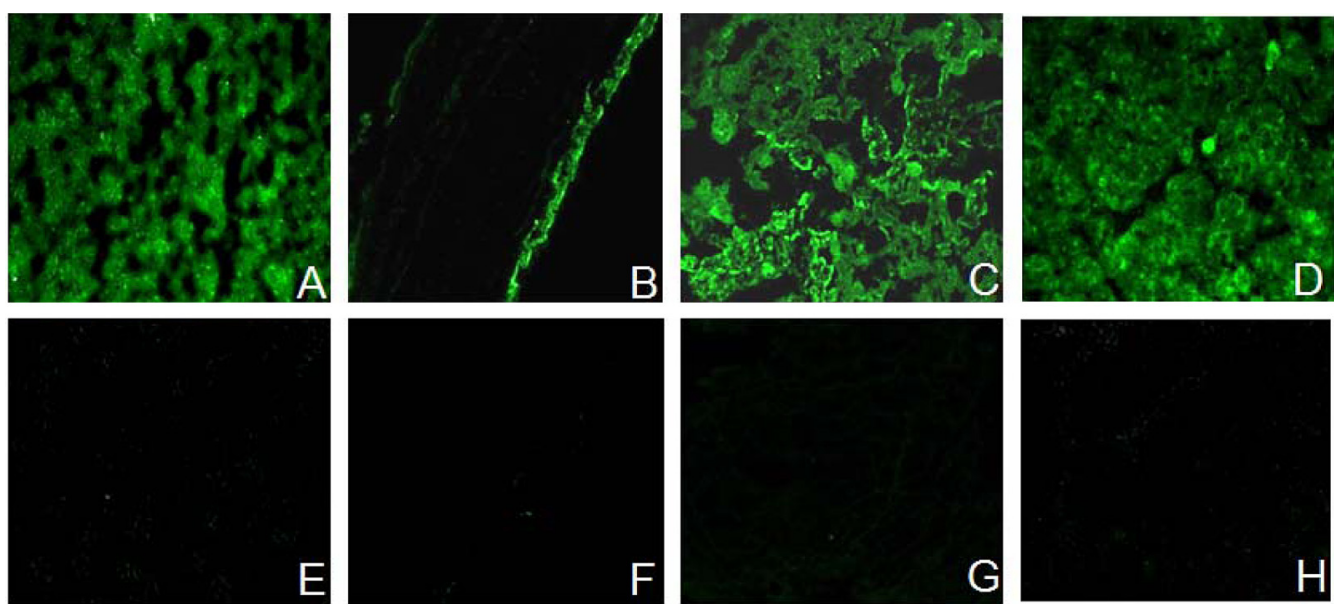

Figure 5. IHC of different organs after 3 DPI with APMV-3 strain Netherlands in turkeys. Viral antigen was visualized by indirect immunofluorescence using polyclonal anti-peptide rabbit antibody specific for the APMV-3 P protein as the primary antibody. The fluorescence was observed in brain (A), trachea (B), lung (C) and pancreas (D) while no fluorescence was observed in organs collected from control birds (E-H). (A color version of this figure is available at www.vetres.org.)

was detected consistently in all the brain, trachea, lung, and pancreas samples that were positive by virus isolation, and no viral antigen was detected in tissue samples that were negative by virus isolation. Extensive immunofluorescence was observed in the brain, trachea, lung and pancreas, suggesting extensive virus replication in these organs (Fig. 5). The intensity of staining was greater for strain Netherlands (Fig. 3) than for strain Wisconsin (data not shown). The presence of fluorescence around the epithelial lining in the lungs and trachea showed the distribution of viral antigens on respiratory epithelium. The presence of generalized fluorescence in the pancreas and brain showed the uniform distribution of viral antigen. However, no viral P antigen was detected in kidney and spleens that were positive by virus isolation, suggesting that replication was not extensive in these organs.

\section{DISCUSSION}

APMV are frequently isolated from wild and domestic birds around the world and have been placed into nine serotypes based on antigenic relatedness. Of these nine serotypes, APMV-1 (NDV) is the best studied. APMV-2 to -9 are present in both free living and domestic birds, but their disease potential is not known in wild birds nor in domestic birds. It is probable that wild birds can transmit APMV-2 to -9 to domestic birds. APMV-2, $-3,-4,-6,-7,-8,-9$ have been isolated from domestic poultry birds and antibodies to these viruses have also been detected in domestic poultry. However, the disease potential of APMV-2 to -9 in chickens and turkeys is mostly unknown.

Here, we have investigated the clinical disease and pathogenicity of APMV-3 strains Netherlands and Wisconsin in embryonated chicken eggs, in 1-day-old chicks inoculated intracerebrally, 1-day-old chicks and turkeys inoculated oculonasally, and in 2-week-old chickens and turkeys inoculated oculonasally. The oculonasal route was intended to resemble a natural route of infection. In this study, 2-week-old chickens and turkeys were chosen over other possible age groups because at this age they are fully susceptible to viral infection. Furthermore, the pathogenicity in 1-day-old chicks and turkeys was studied to assess the impact of APMV-3 infection in young birds. 
APMV-3 strains Netherlands and Wisconsin were first characterized by standard pathogenicity tests (MDT and ICPI). The results of the MDT test showed that APMV-3 strain Netherland was slightly pathogenic (112 h) compared to strain Wisconsin $(>168 \mathrm{~h})$, which was completely apthogenic. Similar findings were also observed in ICPI test: APMV-3 strain Netherland showed an ICPI value of 0.39 , while the ICPI value of strain Wisconsin was zero. Although our results of pathogenicity index tests (MDT and ICPI) showed that APMV-3 strain Netherlands was slightly pathogenic to chickens, both strains belong to the lentogenic group of APMV [7].

Since the APMV-3 strains did not kill 1-dayold chicks by the ICPI test, we investigated whether the absence of neurovirulence was due to lack of virus replication in brain or whether replication occurred without any notable cell destruction. The growth of APMV-3 strains in chicken brain showed that both the strains are equally competent to grow in brain tissue and the highest virus titer was observed $120 \mathrm{~h}$ post inoculation. However, none of the APMV-3 inoculated chicks died 5 DPI, while all chicks inoculated with mesogenic NDV strain BC died 3 DPI. These results suggest that the low ICPI values of APMV-3 strains were due to nondestructive replication of the virus in brain tissue.

It has been shown previously by experimental infection that APMV-3 strain Netherlands is more virulent than strain Wisconsin [3]. In another study, it was shown that the extent of disease and death in the birds after infection with APMV-3 strain Netherlands depends on dose as well as route of infection [4]. In this study, we have evaluated the pathogenicity of APMV-3 strains Netherlands and Wisconsin in 1-dayold and 2-week-old chickens and turkeys by natural (oculonasal) route of infection. The clinical signs of illness were more evident in 1-day-old birds compared to 2-weeks-old birds. In chickens, both the strains of APMV-3 were isolated from tissues of respiratory, digestive and nervous systems. Similar findings were observed in turkeys that were infected with APMV-3 strains. However, the titers of APMV-3 strain Netherlands in different organs was higher than those of strain Wisconsin in all the infected bird groups. Although both the viruses were detected in oral swabs from both 1-day-old and 2-weekold chickens and turkeys, the shedding of viruses were less in 2-week-old birds. These results confirmed previous findings that chickens and turkeys are susceptible to APMV-3 infection and younger birds are more susceptible than older birds. In addition, our results are in agreement with the previous finding that strain Netherland replicates better than strain Wisconsin in chickens and turkeys [4, 32].

Visceral gross lesions were evident in 2-week-old chickens and turkeys at 3 and 5 DPI. Using IHC, viral P protein was detected in the same tissues that were positive by virus isolation except the spleen and kidney tissues that were positive by virus isolation but negative by IHC. It is possible that the virus load in infected spleen and kidney was too low to be detected by IHC. An interesting finding was the presence of large amounts of viral antigens at the epithelial cell linings, suggesting that these cells are highly permissive to APMV-3 replication. Another finding of our IHC study was the presence of viral antigen in both epithelial and sub-epithelial tissues except for trachea where the antigen was localized only in the epithelial surface. This suggests that this virus has a tropism towards both epithelial and sub-epithelial cells. In addition, the detection of viral antigens, and in most cases infectious virus, in multiple internal organs of the birds indicates that both the viruses are capable of replicating in multiple organs rather than being restricted only to respiratory and alimentary tracts. Presumably, the virus reached the various internal organs through the blood stream. Nonetheless, this extensive amount of virus replication was not accompanied by severe disease in birds. These results show that APMV-3 strains are capable of infecting young and adult chickens and turkeys using an oculonasal route of infection. Serologic assays demonstrated a humoral response in all the birds inoculated with either APMV-3 strain, a further indication of successful replication. However, our results suggest that chickens are comparatively more susceptible than turkeys to APMV3 infection.

The F protein cleavage site of NDV is a well characterized determinant of NDV 
pathogenicity in chickens [13, 24, 28]. Virulent NDV strains typically contain a polybasic cleavage site that contains the preferred recognition site for furin (R-X-K/R-R $\downarrow$ ), which is an intracellular protease that is present in most cells. This provides for efficient cleavage in a wide range of tissues, and increases virulence by making it possible for virus to spread systemically. In contrast, avirulent NDV strains typically have basic residues at the -1 and -4 positions relative to the cleavage site and depend on secretory protease (or, in cell culture, added trypsin) for cleavage. Also, the first amino acid of the newly-created F1 terminus is phenylalanine for virulent NDV strains and leucine for avirulent NDV strains, an assignment that also reduces the efficiency of cleavage [25]. The inability to be cleaved by furin limits the replication of avirulent strains to the respiratory and enteric tracts where secretory protease is available for cleavage. The putative $F$ protein cleavage site of APMV-3 strain Netherlands (ARPRGR $\downarrow$ L) and strain Wisconsin (PRPSGR $\downarrow$ L) have 3 or 2 basic residues (underlined), which is similar but not identical to the pattern of virulent and avirulent NDV strains, respectively. Neither APMV-3 strain contains the preferred furin cleavage site. The F1 subunit of both APMV-3 strains begins with a leucine residue, as is characteristic of avirulent NDV strains, rather than a phenylalanine residue, as seen in most virulent NDV strains. The lack of a furin motif in the $\mathrm{F}$ protein cleavage site and the presence of leucine as the first residue at the terminus of the F1 subunit predict that both APMV-3 strains should require added protease for replication in cell culture and should be limited to replication in the respiratory or enteric tracts in vivo. However, APMV-3 strains Netherlands and Wisconsin both replicate well in a wide range of cells in vitro in the absence of added protease, although each strain grows more efficiently (2 $\log _{2}$ higher) with the addition of protease (data not shown). Also, in the present study, both APMV-3 strains were detected abundantly in various internal organs, suggesting a systemic spread of the virus. These results indicate that the structure of the $\mathrm{F}$ protein cleavage site and F1 terminus are poor predictors of the growth properties of APMV-3 in vitro and in vivo. This suggests (i) that the cleavage phenotype of the APMV-3 F protein is determined by factors in addition to the F cleavage site and F1 N-terminus, and (ii) that systemic spread is a multifactorial phenomenon. Furthermore, systemic spread was a poor predictor of pathogenesis, since both APMV-3 strains spread systemically and replicated efficiently in the brain, lung, trachea, and pancreas with little or no disease.

In conclusion, we have shown that 2-weeks old SPF chickens and turkeys are susceptible to APMV-3 infection. However, in commercial chickens and turkeys the disease picture could be quite different depending on management practices, environmental conditions and other concomitant infections. This study has demonstrated that APMV-3 has an affinity for both epithelial as well as sub-epithelial cells of respiratory and alimentary tracts. Finally, dissociations were observed between $F$ protein structure and systemic spread and between systemic spread and disease. Further studies are needed to understand the disease potential of this virus to commercial poultry.

Acknowledgements. We thank Daniel Rockemann and all our laboratory members for their excellent technical assistance and help. This research was supported by NIAID contract No. N01A060009 (85\% support) and NIAID, NIH Intramural Research Program (15\% support). The views expressed herein do not necessarily reflect the official policies of the Department of Health and Human Services; nor does mention of trade names, commercial practices, or organizations imply endorsement by the U.S Government.

\section{REFERENCES}

[1] Alexander D., Paramyxoviridae, Iowa State University Press, Iowa, 2003

[2] Alexander D.J., Chettle N.J., Relationship of parakeet/Netherland/449/75 virus to other avian paramyxovirus, Res. Vet. Sci. (1978) 25:105-106.

[3] Alexander D.J., Avian paramyxoviruses, Vet. Bull. (1980) 50:737-752.

[4] Alexander D.J., Collins M.S., Pathogenicity of PMV-3/parakeet/Netherlands/449/75 for chickens, Avian Pathol. (1982) 11:179-185.

[5] Alexander D.J., Hinshaw V.S., Collins M.S., Yamane N., Characterization of viruses which 
represent further distinct serotypes (PMV-8 and PMV9) of avian paramyxoviruses, Arch. Virol. (1983) 78:29-36.

[6] Alexander D.J., Collins M.S., Characterization of avain paramyxoviruses of serotype PMV-3 isolated from commercial turkey in Great Britain, Avian Pathol. (1984) 13:215-221.

[7] Alexander D.J., Newcastle disease and other avian paramyxoviruses, in: Swayne D.E., Glisson J.R., Jackwood M.W., Pearson J.E., Reed W.M. (Eds.), A laboratory manual for the isolation and identification of avian pathogens, American Association of Avian Pathologists, University of Pennsylvania, Kennett Square, PA, USA, 1998, pp. 156-163.

[8] Anderson C., Kearsley R., Alexander D.J., Russell P.H., Antigenic variation in avian paramyxovirus type 3 isolates detected by mouse monoclonal antibodies, Avian Pathol. (1987) 16:691-698.

[9] Anderson C.L., Russell P.H., Monoclonal antibodies against avian paramyxovirus-3: antigenic mapping and functional analysis of the haemagglutinin-neuraminidase protein and the characterization of nonspecific monoclonal antibodies, Arch. Virol. (1988) 101:49-63.

[10] Beck I., Gerlach H., Burkhardt E., Kaleta E.F., Investigation of several selected adjuvants regarding their efficacy and side effects for the production of a vaccine for parakeets to prevent a disease caused by a paramyxovirus type 3, Vaccine (2003) 21:1006-1022.

[11] Capua I., De Nardi R., Beato M.S., Terregino C., Scremin M., Guberti V., Isolation of an avian paramyxovirus type 9 from migratory waterfowl in Italy, Vet. Rec. (2004) 155:156.

[12] Chang P.C., Hsieh M.L., Shien J.H., Graham D.A., Lee M.S., Shieh H.K., Complete nucleotide sequence of avian paramyxovirus type 6 isolated from ducks, J. Gen. Virol. (2001) 82:2157-2168.

[13] De Leeuw O., Peeters B., Complete nucleotide sequence of Newcastle disease virus: evidence for the existence of a new genus within the subfamily Paramyxovirinae, J. Gen. Virol. (1999) 80:131-136.

[14] Gough R.E., Alexander D.J., Avian paramyxovirus type 4 isolated from a ringed teal (Calonetta leucophrys), Vet. Rec. (1984) 115:653.

[15] Huang Z., Panda A., Elankumaran S., Govindarajan D., Rockemann D.D., Samal S.K., The hemagglutinin-neuraminidase protein of Newcastle disease virus determines tropism and virulence, J. Virol. (2004) 78:4176-4184.

[16] Kaleta E.F., Werner O., Hemberger Y., Isolation and characterization of avian paramyxovirus type $3 \mathrm{~b}$ from farmed Namibian ostriches (Struthio camelus f. dom.), Berl. Munch. Tierarztl. Wochenschr. (2010) 123:103-110.

[17] Krishnamurthy S., Samal S.K., Nucleotide sequences of the trailer, nucleocapsid protein gene and intergenic regions of Newcastle disease virus strain Beaudette $\mathrm{C}$ and completion of the entire genome sequence, J. Gen. Virol. (1998) 79:24192424 .

[18] Kumar S., Nayak B., Collins P.L., Samal S.K., Complete genome sequence of avian paramyxovirus type 3 reveals an unusually long trailer region, Virus Res. (2008) 137:189-197.

[19] Kumar S., Nayak B., Samuel A.S., Xiao S., Collins P.L., Samal S.K., Complete genome sequence of avian paramyxovirus-3 strain Wisconsin: evidence for the existence of subgroups within the serotype, Virus Res. (2010) 149:78-85.

[20] Lamb R., Parks G., Paramyxoviridae: the viruses and their replication, Lippincott Williams \& Wilkins, Philadelphia, 2007.

[21] Macpherson I., Watt R.G., Alexander D.J., Isolation of avian paramyxovirus other than Newcastle disease virus from commercial poultry in Great Britain, Vet. Rec. (1983) 112:479-480.

[22] Maldonado A., Arenas A., Tarradas M.C., Luque I., Astorga R., Perea J.A., Miranda A., Serological survey for avian paramyxoviruses from wildfowl in aquatic habitats in Andalusia, J. Wildl. Dis. (1995) 31:66-69.

[23] Mayo M.A., A summary of taxonomic changes recently approved by ICTV, Arch. Virol. (2002) 147:1655-1663.

[24] Millar N.S., Chambers P., Emmerson P.T., Nucleotide sequence of the fusion and haemagglutininneuraminidase glycoprotein genes of Newcastle disease virus, strain Ulster: molecular basis for variations in pathogenicity between strains, J. Gen. Virol. (1988) 69:613-620.

[25] Morrison T., McQuain C., Sergel T., McGinnes L., Reitter J., The role of the amino terminus of F1 of the Newcastle disease virus fusion protein in cleavage and fusion, Virology (1993) 193:997-1000.

[26] Nayak B., Kumar S., Collins P.L., Samal S.K., Molecular characterization and complete genome sequence of avian paramyxovirus type 4 prototype strain duck/Hong Kong/D3/75, Virol. J. (2008) 5:124.

[27] Paldurai A., Subbiah M., Kumar S., Collins P.L., Samal S.K., Complete genome sequences of avian paramyxovirus type 8 strains goose/Delaware/1053/76 and pintail/Wakuya/20/78, Virus Res. (2009) 142:144153. 
[28] Panda A., Huang Z., Elankumaran S., Rockemann D.D., Samal S.K., Role of fusion protein cleavage site in the virulence of Newcastle disease virus, Microb. Pathog. (2004) 36:1-10.

[29] Redmann T., Zeydanli M.M., Herbst W., Kaleta E.F., Isolation of a paramyxovirus-3 from turkeys with respiratory tract disease in Germany, Dtsch. Tierarztl. Wochenschr. (1991) 98:138-141 (in German).

[30] Reed L.J., Muench H., A simple method of estimation of $50 \%$ end points, Am. J. Hyg. (1938) 27:493-497.

[31] Rout S.N., Samal S.K., The large polymerase protein is associated with the virulence of Newcastle disease virus, J. Virol. (2008) 82:7828-7836.

[32] Russell P.H., Awang I.P., Parsons G., Alexander D.J., Pathogenicity of PMV-3/Turkey/England/Midland poultry holdings/1981 for chickens and turkeys, Avian Pathol. (1989) 18:125-134.

[33] Saif Y.M., Mohan R., Ward L., Senne D.A., Panigrahy B., Dearth R.N., Natural and experimental infection of turkeys with avian paramyxovirus-7, Avian Dis. (1997) 41:326-329.

[34] Samuel A.S., Kumar S., Madhuri S., Collins P.L., Samal S.K., Complete sequence of the genome of avian paramyxovirus type 9 and comparison with other paramyxoviruses, Virus Res. (2009) 142:10-18.

[35] Shortridge K.F., Alexander D.J., Collins M.S., Isolation and properties of viruses from poultry in Hong Kong which represent a new (sixth) distinct group of avian paramyxoviruses, J. Gen. Virol. (1980) 49:255-262.
[36] Smit T., Rondhuis P.R., Studies on a virus isolated from the brain of a parakeet (Neophema sp), Avian Pathol. (1976) 5:21-30.

[37] Stallknecht D.E., Senne D.A., Zwank P.J., Shane S.M., Kearney M.T., Avian paramyxoviruses from migrating and resident ducks in coastal Louisiana, J. Wildl. Dis. (1991) 27:123-128.

[38] Subbiah M., Xiao S., Collins P.L., Samal S.K., Complete sequence of the genome of avian paramyxovirus type 2 (strain Yucaipa) and comparison with other paramyxoviruses, Virus Res. (2008) 137:40-48.

[39] Tumova B., Robinson J.H., Easterday B.C., A hitherto unreported paramyxovirus of turkeys, Res. Vet. Sci. (1979) 27:135-140.

[40] Wakamatsu N., King D.J., Kapczynski D.R., Seal B.S., Brown C.C., Experimental pathogenesis for chickens, turkeys, and pigeons of exotic Newcastle disease virus from an outbreak in California during 2002-2003, Vet. Pathol. (2006) 43:925-933.

[41] Warke A., Stallknecht D., Williams S.M., Pritchard N., Mundt E., Comparative study on the pathogenicity and immunogenicity of wild bird isolates of avian paramyxovirus 2, 4, and 6 in chickens, Avian Pathol. (2008) 37:429-434.

[42] Xiao S., Paldurai A., Nayak B., Subbiah M., Collins P.L., Samal S.K., Complete genome sequence of avian paramyxovirus type 7 (strain Tennessee) and comparison with other paramyxoviruses, Virus Res. (2009) 145:80-91. 